\title{
A Mathematical Model of HIV and Malaria Co-Infection in Sub-Saharan Africa
}

Kamal Barley ${ }^{1}$, David Murillo', Svetlana Roudenko', Ana M. Tameru ${ }^{2 *}$ and SharquettaTatum ${ }^{3}$

${ }^{1}$ Department of Mathematics and Statistics, Arizona State University, Tempe, AZ 85287, USA

${ }^{2}$ Department of Mathematics and Computer Science, Alabama State University, Montgomery, AL 36101, USA

${ }^{3}$ Department of Mathematics, Alabama A \& M University, Normal, AL 35762, USA

\begin{abstract}
Malaria and HIV are two of the most deadly diseases in Africa. Combined they account for 4 million deaths each year, and according to the Center for Disease Control and Prevention (CDC), there is an estimated 5 percent increase in malaria deaths in those who tested positive for HIV than those without HIV infection. Since the coinfections were recorded, malaria has seen a 28 percent increase in its incidence. These results raise the possibility that biological differences could alter the effect of co-infection and underscore the importance of identifying these factors for the implementation of control interventions focused on co-infection. Malaria associated death rates have nearly doubled for those with co-infections. The biological integrations between the malaria parasite and HIV are not fully understood, but it is conceivable that the parasite or viral load can increase by an order of magnitude due to coinfection. HIV-infected persons are at increased risk for clinical malaria; the risk is greatest when immune suppression is advanced. Malaria is associated with increases in HIV viral load that, while modest, may impact HIV progression or the risk of HIV transmission. We also showed that in the Full Model, total cause of deaths are from co-infection when the amplification factor $\rho_{i,}, i=1,2,3,4$, is larger than 25 . We introduce a system of differential equations linking the host-vector system of malaria with co-infection with HIV. Data were collected from Sub-Saharan Africa for the global parameter estimates and we simulated for sensitivity analysis using data collected from Malawi. Finally, these simulations show that the HIV-induced increase in susceptibility to malaria infection has marginal effect on the new cases of HIV and malaria but increases the number of new cases of the dual HIV-malaria infection.
\end{abstract}

Keywords: Mathematical Biology; Computational Biology

\section{Introduction}

The Sub-Saharan region of Africa has many endemic diseases including malaria and HIV, which are two of the deadliest diseases of our time [1]. The geographic overlap of these diseases (Figures 1 and 2) in Sub-Saharan Africa facilitates co-infections with HIV and malaria [2]. Since both diseases are endemic and the length of infection for both diseases can be several years, the burden of co-infection is a real and pressing problem.

Malaria is an old disease that was first studied by Ross in the late 1800 's [3,4]. Despite over 100 years of study and advanced biological, medical, and mathematical understanding, we have yet to come to a viable solution for this disease that has already killed hundreds of millions of individuals. HIV/AIDS, by contrast, is a relatively new disease that has only been studied since the 1980's. Like malaria, HIV has received considerable attention from the scientific community and continues to kill millions while we search for a cure. While AIDS (last HIV stage) is characterized by the process of opportunistic infections, malaria is not typical in this regard. The co-infection between HIV/ AIDS and malaria is not well understood. It is our hope that through our model the joint effects of co-infections are better understood.

The prevalence of HIV in the Sub-Saharan region is less than $20 \%$ for all countries except Botswana, Lesotho, and Swaziland. Malaria increases the viral load in HIV patients but this effect may be reversed with malaria treatment [1] and on the other hand, HIV increases the risk to be infected by malaria because of the weakness of the immune system [5]. Because of the increase in viral loads in HIV patients from malaria, HIV transmission is thought to become twice as likely to be passed on to a noninfected individual [2].

In this study we propose a mathematical model for the joint dynamics of HIV and malaria co-infections. Our model is given by a set of six differential equations (which we later reduce to four). The details of the co-infection are very complicated, yet, we have managed to model the effects of co-infections in a simple setting (a detailed discussion is deferred to Section 2). The remainder of the paper is organized as follows: below we give a brief discussion of HIV/AIDS and malaria. In Section 2 we analyze the stability of our model and find the basic reproductive number of our model, using the next generator operator approach. In Section 3, we discuss some simplifying assumptions, reduce our model to a system of four equations, and carry out the corresponding stability analysis. In Section 4, we examine the model in the absence of malaria and also in the absence of HIV. In Section 5 , we discuss our conclusions, list avenues for potential future work. Finally we include mathematical derivations of $R_{0}$ and Matlab code in the Appendix.

\section{HIV/AIDS}

HIV has killed an estimated 25 million individuals worldwide [6]. Since it was discoveredin 1981, HIV has become one of the leading causes of death, globally, affecting mostlyimpoverished people already suffering from poor nutrition and health [2]. HIV standsfor human

*Corresponding author: Ana M. Tameru, Department of Mathematics and Computer Science, Alabama State University, 915 Jackson St, Montgomery, AL 36101, USA, Tel: 334-229-6828; E-mail: atameru@alasu.edu

Received July 10, 2012; Accepted September 04, 2012; Published Septembe 14,2012

Citation: Barley K, Murillo D, Roudenko S, Tameru AM, Tatum S (2012) A Mathematical Model of HIV and Malaria Co-Infection in Sub-Saharan Africa. J AIDS Clinic Res 3:173. doi:10.4172/2155-6113.1000173

Copyright: (C) 2012 Barley K, et al. This is an open-access article distributed unde the terms of the Creative Commons Attribution License, which permits unrestricted use, distribution, and reproduction in any medium, provided the original author and source are credited. 


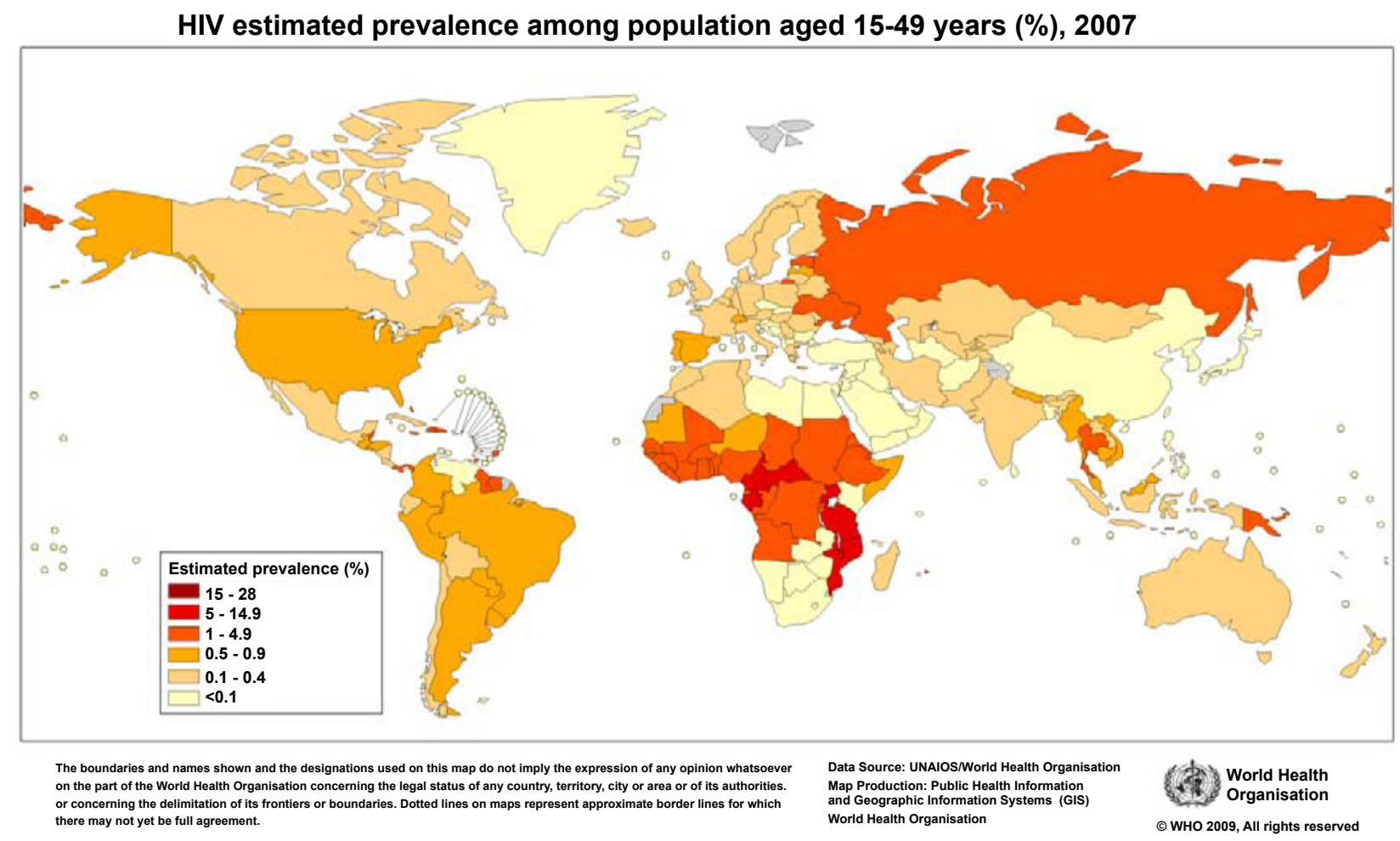

Figure 1: Global Prevalence of HIV according to WHO in 2009 [12]

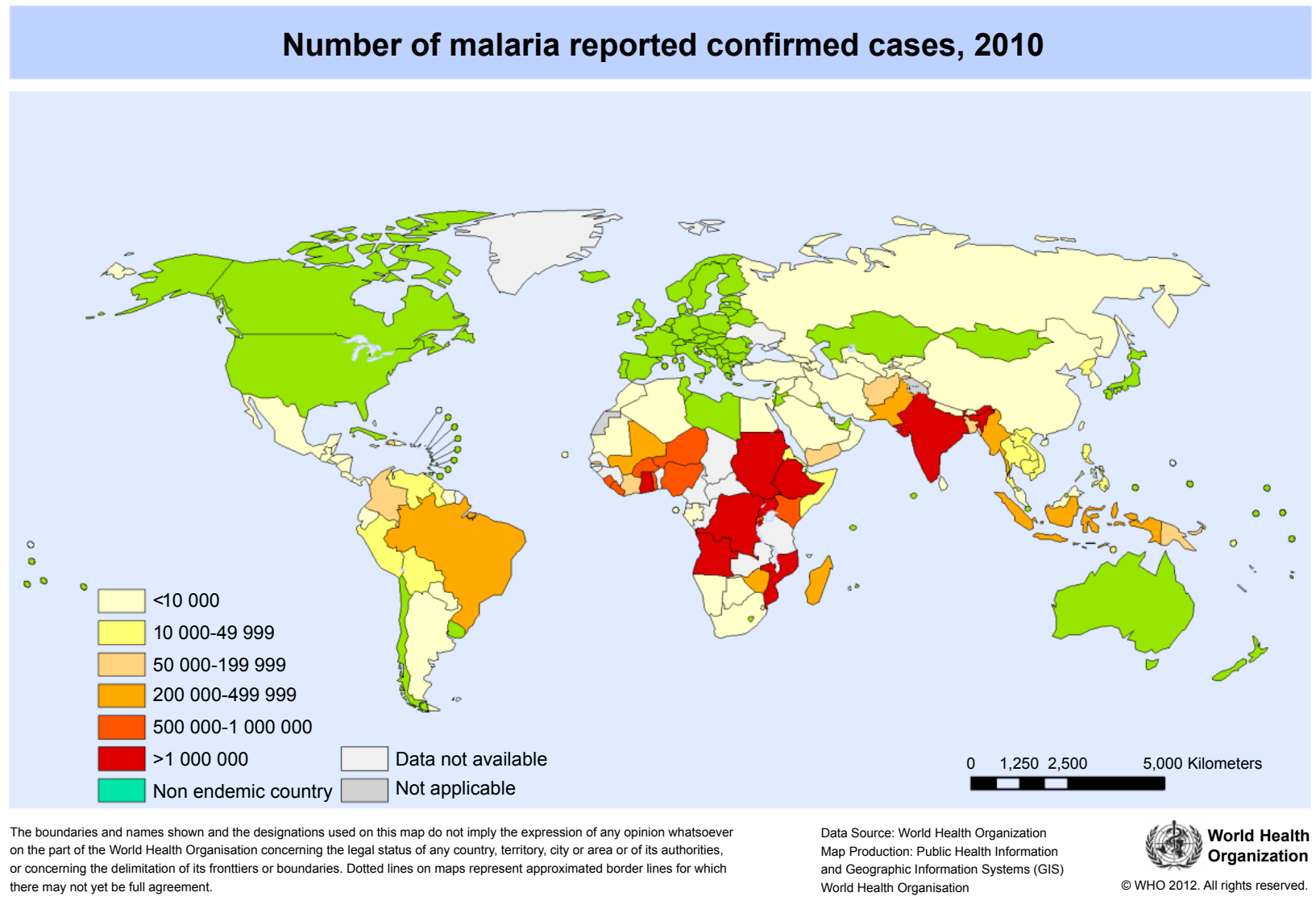

Figure 2: Global Prevalence of Malaria according to WHO in 2012 [10]. 
immunodeficiency virus; it is a virus that attacks the immune system. WhileHIV does not kill, it causes the immune system to become defenseless against otheropportunistic diseases it could normally fight off. An estimated 25 million people areinfected with HIV each year in Africa [2].

\section{Malaria}

According to the CDC, malaria was first discovered centuries ago by the Chinese in 2700 BC. However it was in the late 1800's when Ross made his ground breaking discoveries that led to our understanding of the mechanics behind malaria infection [7]. Malaria is a mosquito borne disease and kills about 1 to 2 million people a year, of which most are children [8]. If left untreated malaria attacks the liver and moves through the bloodstream infecting every organ it can until the body shuts down leading to death. In Africa an estimated 350 million individuals are infected with the disease [6]. Although malaria is treatable, the drugs can be too expensive or too difficult to distribute to the general public in countries where it is endemic. Like HIV it affects mostly impoverished people and, like HIV, it is a contributor to the impoverishment of many countries in Sub-Saharan Africa.

\section{Full Model}

There are many challenges in the derivation of an HIV/malaria coinfection model. HIV has many methods of transmission; the principles being: heterosexual and homosexual contact, intravenous needle sharing and mother to child transmission. The age group most affected by each method of transmission varies widely. Malaria is transmitted by a vector (mosquito), but the exact species varies from region to region. Mostly children die from malaria. For simplification we assume that our susceptible population is the general population that is at risk to getting an HIV infection at a rate proportional to the density of HIV infected people. Similarly, our susceptible population is also assumed to be at risk to get malaria at a rate proportional to the density of infected mosquitoes. We divide the total human population, $N$, into 4 different classes: $S$, represents the susceptible class; $I_{M}$, represents infectious malaria class; $I_{H}$, represents infectious HIV class; $I_{H M}$, represents infectious with both HIV and malaria class; the total mosquito population, $N_{V}$, is divided into 2 different classes: $\mathrm{V}$, represents the susceptible vector class; and $I_{V}$, represents the infectious vector class.

It is known that there is an incubation period for malaria [6], but since we are interested in long term dynamics we ignore any latent or exposed classes. We also assume the total vector population is constant, but since death is a major concern for people infected with HIV or malaria, we do include disease induced mortality for people. Thus, the human population is not assumed to be constant, in fact Malawi has an estimated growth rate of 2.76 percent. Instead we assume a constant recruitment rate in the $S$ class. We also assume susceptible people cannot simultaneously get infected with malaria and HIV since the transmission mechanics are completely different for the two diseases. To get to the $I_{H M}$ class a person must first enter either the $I_{H}$ or the $I_{M}$ class. However, a person in the $I_{H M}$ class can transmit both diseases. Furthermore, since a person's immune system is compromised, that person has a higher probability of transmission given a "contact" has occurred. Here a "contact" is any process that can transmit an infection. We model this with an amplification factor $\rho_{i}$, where $i$ depend on classes involved in the transmission.

We arrive at the following system of equations for the Full Model:

$$
\begin{aligned}
& \frac{d S(t)}{d t}=\wedge-\left(\frac{\beta_{V M} I_{V}(t)}{N_{V}}+\frac{\beta_{H} I_{H}(t)}{N}+\frac{\rho_{1} \beta_{H} I_{H M}(t)}{N}+\alpha\right) S(t)+\gamma I_{M}(t) \\
& \frac{d I_{M}(t)}{d t}=\frac{\beta_{V M} I_{V}(t)}{N_{V}} S(t)-\left(\frac{\beta_{H} I_{H}(t)}{N}+\frac{\rho_{2} \beta_{H} I_{H M}(t)}{N}+\gamma+\mu_{M}+\alpha\right) I_{M}(t) \\
& \frac{d I_{H M}(t)}{d t}=\left(\frac{\beta_{H} I_{M}(t)}{N}+\frac{\rho_{3} \beta_{V M} I_{V}(t)}{N_{V}}\right) I_{H}(t)+\left(\frac{\rho_{2} \beta_{H} I_{M}(t)}{N}-\gamma K-\mu_{H M}-\alpha\right) I_{H M}(t) \\
& \frac{d I_{H}(t)}{d t}=\left(\frac{\beta_{H} S(t)}{N}+\frac{\rho_{3} \beta_{V M} I_{V}(t)}{N_{V}}-\mu_{H}-\alpha\right) I_{H}(t)+\left(\frac{\rho_{1} \beta_{H} S(t)}{N}-\gamma K\right) I_{H M}(t) \\
& \frac{d V(t)}{d t}=\mu_{V} N_{V}-\left(\frac{\beta_{M V} I_{M}(t)}{N}+\frac{\rho_{4} \beta_{M V} I_{H M}(t)}{N}+\mu_{V}\right) V(t) \\
& \frac{d I_{V}(t)}{d t}=\left(\frac{\beta_{M V} I_{M}(t)}{N}+\frac{\rho_{4} \beta_{M V} I_{H M}(t)}{N}\right) V(t)-\mu_{V} I_{V}(t)
\end{aligned}
$$

Where parameter definitions are given in Table 1, note that the rates relating to the human population have been rescaled by the initial total population for numerical stability (Figure 3 ).

\section{Local stability of the full model}

The disease free equilibrium $(D F E)$ is straight forward to calculate by setting the infectious classes $\left(I_{M}, I_{H}, I_{H M}, I_{V}\right)$ equal to zero:

$$
D F E=\left(S^{o}, I_{M}^{o}, I_{H}^{o}, I_{H M}^{o}, V^{o}, I_{V}^{o}\right)=\left(\frac{\wedge}{\alpha}, 0,0,0, N_{V}, 0\right)
$$

This implies that the population, in the absence of diseases, will reach a demographic equilibrium. It remains to study the stability of this equilibrium point.

The basic reproductive number represents the average number of secondary infections caused by a "typical" infectious individual in a mostly susceptible population. It is the threshold parameter that usually determines the stability of the DFE. We use the next generation operator approach [9] to arrive at the following $R_{0}$

$$
R_{0}=\max \left\{R_{0 H}, R_{0 M}\right\}
$$

where

\begin{tabular}{|l|l|c|c|}
\hline Parameter & Definition & Malawi & Sub-Saharan \\
Africa
\end{tabular}




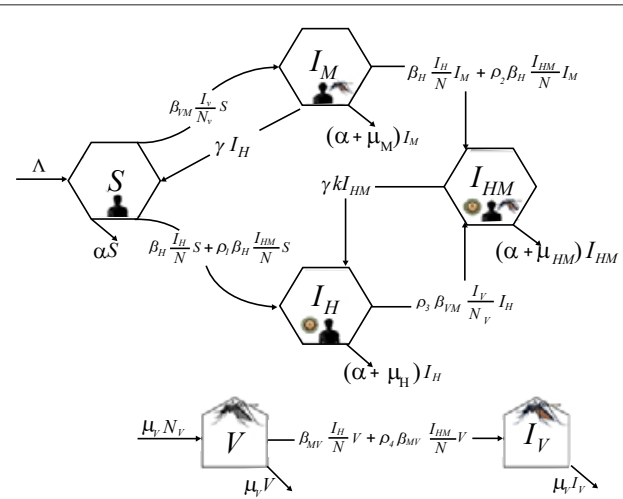

Figure 3: Full Model. Note there are two modes of transmission from the classes $I_{M}$ to $I_{H M}, S$ to $I_{H}$, and $V$ to $I_{V}$.

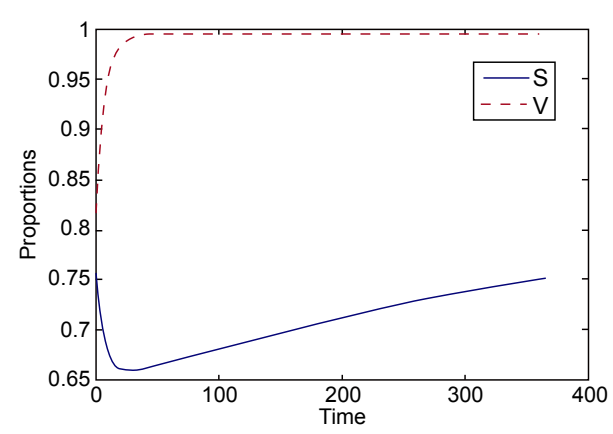

Figure 4: The dynamics of the vectors are on a much faster time scale than the dynamics of the humans.

$$
\begin{aligned}
& R_{O H}=\frac{\beta_{H}}{\mu_{H}+\alpha} \\
& \text { and } \\
& R_{O M}=\sqrt{\frac{\beta_{M V} \beta_{V M}}{\mu_{V}\left(\mu_{H}+\lambda+\alpha\right)}}
\end{aligned}
$$

A formal proof is deferred to the Appendix. $R_{O H}$ represents the rate at which HIV is transmitted $\left(\beta_{H}\right)$ times the average time spent in the HIV class $\left(\frac{1}{\mu H+a}\right) \cdot R_{O M}$, represents the square root of the transmission rate from human to vector $\left(\beta_{M V}\right)$ times the average time spent in the infectious vector class $\left(\frac{1}{\mu V}\right)$ times the transmission rate from vector to human $\left(\beta_{r M}\right)$ times the average time spent in the infectious malaria $\operatorname{class}\left(\frac{1}{\mu M+\gamma+\alpha}\right)$. There is a square root in this term because malaria is a two-stepprocess; meaning for an infected individual to infect another individual a mosquito must transmit the disease.

We then arrive at the following theorem: If $R_{0}<1$, the DFE is locally asymptotically stable. The DFE of the Full Model is unstable if $R_{0}>1$ see Appendix for a proof.

We remind the reader that our goal is to understand the dynamics of HIV and malaria co-infections using the simplest possible model. While $R_{0}$ gives us insight, physical intuition, and the numerical solutions indicate there should be a Co-Infection Equilibrium (CE). Unfortunately, our model is too complicated to arrive at an explicit solution for the CE. Previous work [6] and numerical solution (Figure 4) pose a possible answer: the mosquito population is on a fast time scale relative to the dynamics of the human population. We use this difference in time scale to simplify our model.

\section{Reduced Model}

We reduce our full model to a system of 4 nonlinear equations as follows: First we note that the birth rate going into the vector classes is equal to the mortality rate going out of them, that is the total vector population is constant. Hence, we set $V=N_{V}-I_{V}$. Furthermore, we assume that the vector dynamics are fast relative to the human dynamics, allowing us to make the pseudo steady state approximation. That is we assume that the vector system is at a steady state and substitute for $V$ to get:

$$
I_{V}^{*}=\frac{\beta_{M V} N_{V}\left(I_{M}+\rho_{4} I_{H M}\right)}{\beta_{M V} I_{M}+\rho_{4} \beta_{M V} I_{H M}+\mu_{V} N}
$$

Where $I_{V}{ }^{*}$ is the equilibrium value of the $I_{V}$ class, this is simply a rational function of $I_{M}$ and $I_{H M}$. Using the fact that the vector dynamics go a lot faster than human dynamics lead to the following reduced model (Figure 5):

$$
\begin{aligned}
& \frac{d S(t)}{d t}=\wedge-\left(\frac{\beta_{V M} \beta_{M V}\left(I_{M}+\rho_{4} I_{H M}\right)}{\beta_{M V} I_{M}+\rho_{4} \beta_{M V} I_{H M}+\mu_{V} N}+\frac{\beta_{H} I_{H}+\rho_{1} \beta_{H} I_{H M}}{N}+\alpha\right) S+\gamma I_{M} \\
& \frac{d I_{M}(t)}{d t}=\frac{\beta_{V M} \beta_{M V}\left(I_{M}+\rho_{4} I_{H M}\right)}{\beta_{M V} I_{M}+\rho_{4} \beta_{M V} I_{H M}+\mu_{V} N} S-\left(\frac{\beta_{H} I_{H}+\rho_{2} \beta_{H} I_{H M}}{N}+\mu_{M}+\gamma+\alpha\right) I_{M} \\
& \frac{d I_{H}(t)}{d t}=\left(\frac{\beta_{H} S}{N}-\frac{\rho_{3} \beta_{V M} \beta_{M V}\left(I_{M}+\rho_{4} I_{H M}\right)}{\beta_{M V} I_{M}+\rho_{4} \beta_{M V} I_{H M}+\mu_{V} N}-\mu_{H}-\alpha\right) I_{H}+\left(\frac{\rho_{1} \beta_{H} S}{N}+K \gamma\right) I_{H M} \\
& \frac{d I_{H M}(t)}{d t}=\left(\frac{\beta_{H} I_{M}}{N}+\frac{\rho_{3} \beta_{V M} \beta_{M V}\left(I_{M}+\rho_{4} I_{H M}\right)}{\beta_{M V} I_{M}+\rho_{4} \beta_{M V} I_{H M}+\mu_{V} N}\right) I_{H}+\left(\frac{\rho_{1} \beta_{H} I_{M}}{N}-\mu_{H M}-K \gamma-\alpha\right) I_{H M}
\end{aligned}
$$

\section{Local stability of the reduced model}

The Disease Free Equilibrium of the Reduced Model (DFER) can be derived from the DFE and carries the analogous interpretation.

$\operatorname{DFER}=\left(S^{0}, I_{M}^{0}, I_{H}^{0}, I_{H M}^{0}\right)=\left(\frac{\wedge}{\alpha}, 0,0,0\right)$

Similar the stability analysis and $R_{0}$ calculations follow directly from that of the Full Model.

$R_{0}=\max \left\{R_{0 H}, R_{0 M}\right\}$

where

$R_{O H}=\frac{\beta_{H}}{\mu_{H}+\alpha}$

and

$R_{O M}=\sqrt{\frac{\beta_{M V} \beta_{V M}}{\mu_{V}\left(\mu_{H}+\gamma+\alpha\right)}}$

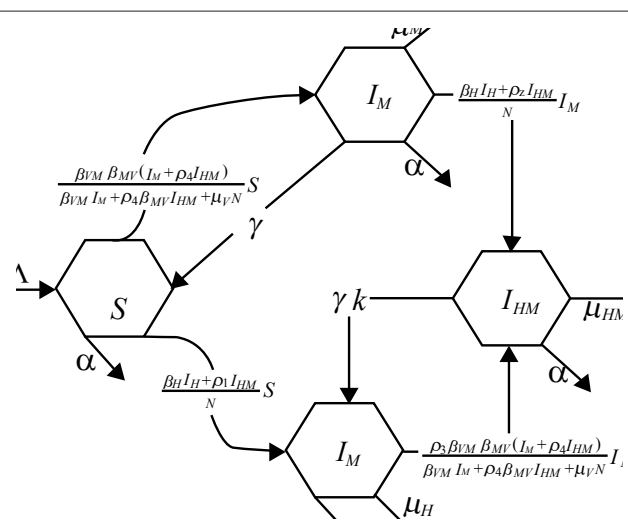

Figure 5: Reduced Model. The vector population is assumed to reach its equilibrium much faster than the human population. 


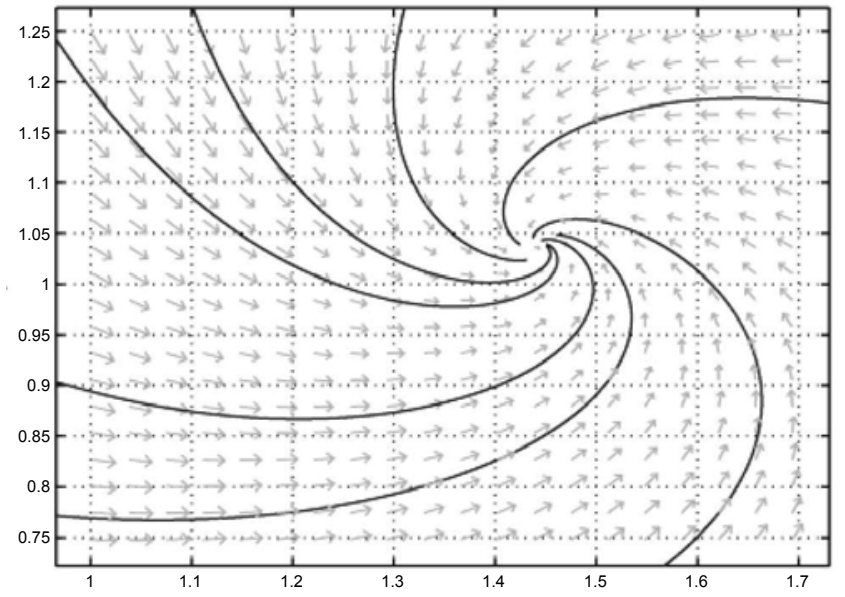

Figure 6: $S^{\prime}$ vsl' for Malawi, where $\Lambda=0.00039, \mu_{H}=0.00023, \beta_{H}=0.0005, \alpha$ $=0.000060883$.

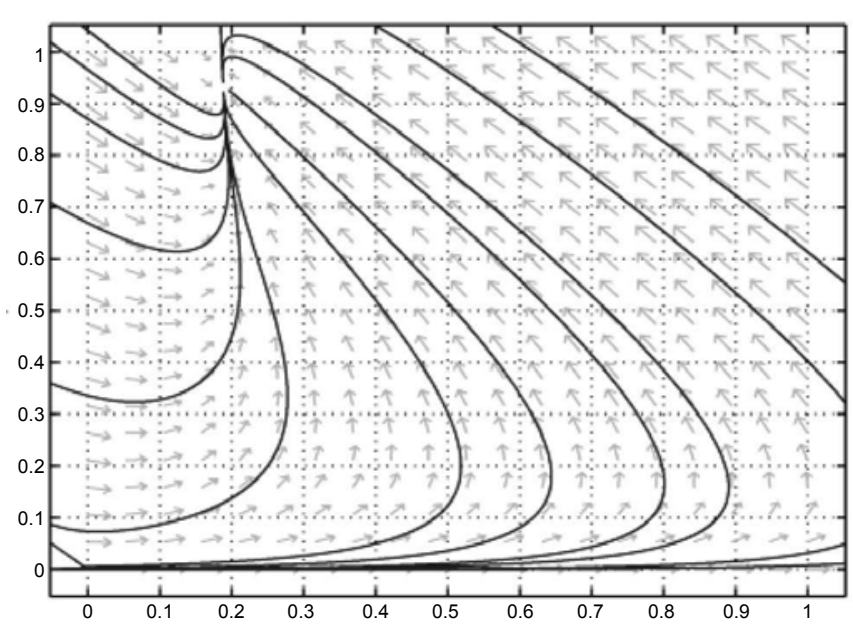

Figure 7: S'vsl' for Malawi, where $\Lambda=0.00039, \mu_{v}=0.1429, \mu_{M}=0.0003454$, $\beta_{V M}=0.12, \beta_{M V}=0.003, \alpha=0.000060883, y=0.00001, N=S+I_{M}$.

Where $R_{O H}$ and $R_{O M}$ have the same biological interpretations as before. It was our hope that the reduced model would lend itself to an analytical calculation of the coexistence equilibrium point. However, even with the use of a computer algebra system we were unable to get an analytical form for it. Instead we employ numerical solutions and single disease models to gain insight into our problem of co-infection.

\section{Single Disease Vs Co-Infection}

To evaluate the effects of co-infection in our model we look at the case of only a single disease for comparison. The HIV only model is a simple SI model obtained by setting the infectious malaria classes ( $I_{M}, I_{H M}$ and $I_{V}$ ) to zero. The dynamics of this model are known, the DFE is stable when $R_{O H}<1$ and there is a stable HIV only endemic equilibrium when $R_{O H}>1$ [9]. Figure 6 is the phase portrait of the HIV only model with the parameters from Malawi. Similarly the malaria only model is obtained by setting $I_{H}$ and $I_{H M}$ to zero. It is a vector-host SI model with essentially the same dynamics as the SI model. Figure 7 shows the phase portrait of the malaria only model with the parameters from Malawi.

\section{Comparison with co-infection: mortality}

HIV Insite estimates that additional mortality due to co-infection may increase by less than 5 percent to 118 percent. Figure 8 compares the total deaths calculated from that HIV only model, malaria only model, and Full Model. These deaths are calculated with varying $\rho$ where $\rho_{i}=\rho$ for $i=1,2,3,4$, we make the assumption that all the $\left(\rho_{i}\right)^{\prime} s$ are equal for simplicity. Since the HIV and malaria only models do not have any co-infections, they are constant with respect to $\rho$. With $\rho=$ 1 (there is no additional infectivity due to co-infection) the increased deaths due to co-infection was approximately 3 percent and with $\rho$ at approximately thirty, the number of deaths double, agreeing with the HIV Insite estimate.

Since it is not known what the additional infectivity due to coinfection is, we plotted the diseases induced deaths $V S \rho$ in Figure 9. For $\rho$ small there was very little increased mortality, but if $\rho$ was larger than 25 then co-infection deaths dominate total deaths.

\section{Sensitivity analysis}

Getting reliable data is a ubiquitous problem in mathematical biology. While we were able to find many of the parameters in Table 1 , some parameters were estimated. Thus we would hope our estimate of $R_{0}$ is not very sensitive to parameter values. We perform a sensitivity analysis on $R_{0}$ with respect to our parameters [10]. The sensitivity index $S$ is defined as:

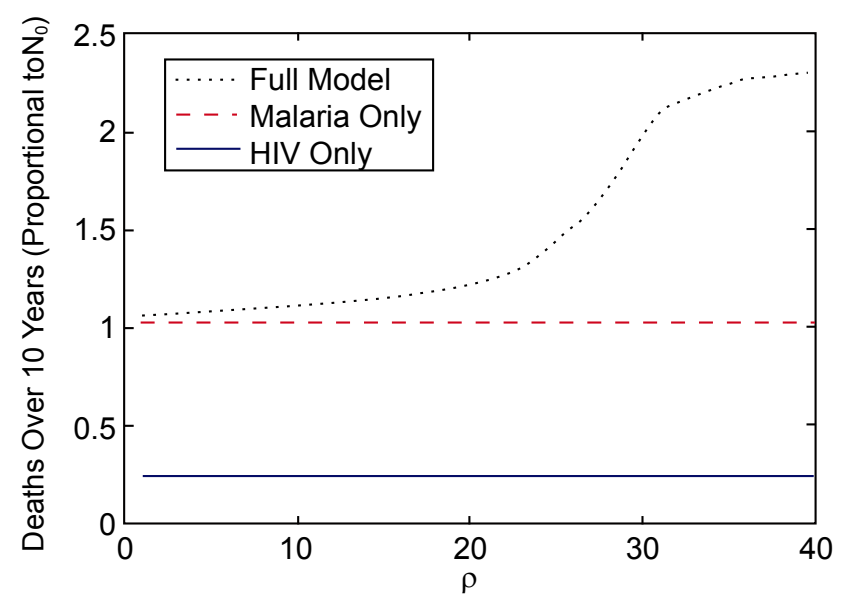

Figure 8: Death Due to Diseases.

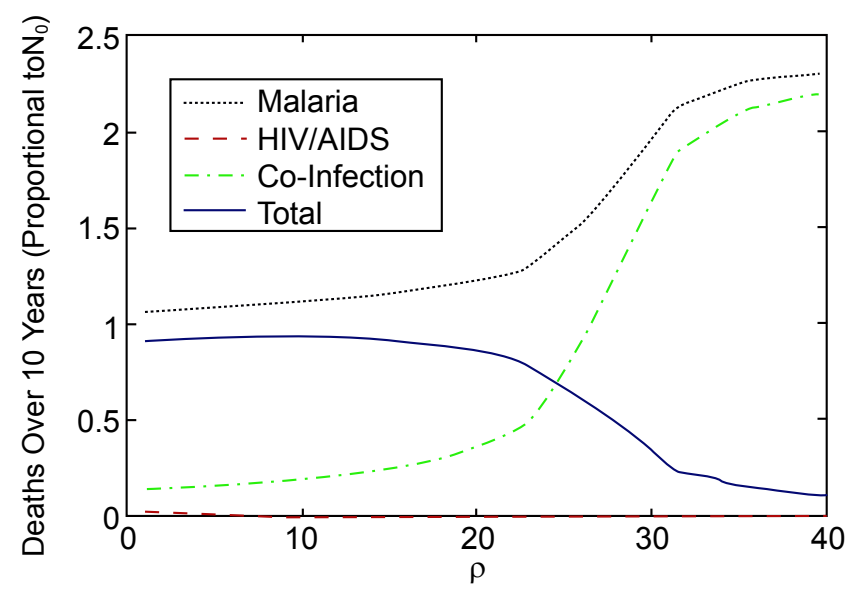

Figure 9: Total deaths are dominated by co-infection deaths when $\rho$ is larger than 25 in the Full Model. 


\begin{tabular}{|c|c|c|c|c|}
\hline$R_{O}$ & Parameter & $\frac{\partial R_{0}}{\partial \text { parameter }} \frac{\text { parameter }}{R_{0}}$ & Values for Malawi & $\begin{array}{c}\text { Values for Sub- } \\
\text { Saharan } \\
\text { Africa }\end{array}$ \\
\hline$R_{O H}$ & $\beta_{H}$ & 1 & 1 & 1 \\
$R_{O H}$ & $\mu_{H}$ & $-\frac{\mu_{H}}{\mu_{H}+\alpha}$ & -0.6969230846 & -0.8011759871 \\
$R_{O H}$ & $\alpha_{H}$ & $-\frac{\alpha}{\mu_{H_{n}}+\alpha}$ & -0.3030769154 & -0.1988240130 \\
$R_{O M}$ & $\beta_{M V}$ & $\frac{1}{2}$ & $\frac{1}{2}$ & $\frac{1}{2}$ \\
$R_{O M}$ & $\beta_{V M}$ & $\frac{1}{2}$ & $\frac{1}{2}$ & $\frac{1}{2}$ \\
$R_{O M}$ & $\mu_{V}$ & $-\frac{1}{2} \times \frac{1}{\mu_{\mu}}$ & $-\frac{1}{2}$ & $-\frac{1}{2}$ \\
$R_{O M}$ & $\mu_{M}$ & $-\frac{1}{2} \times \frac{\gamma}{\mu_{M}+\gamma+\alpha}$ & -0.2631486722 & -0.03384629978 \\
$R_{O M}$ & $\gamma$ & $-\frac{1}{2} \times \frac{\alpha}{\mu_{M}+\gamma+\alpha}$ & -0.1904666128 & -0.4605605355 \\
$R_{O M}$ & $\alpha$ & -0.04638471514 & -0.005593164735 \\
\hline
\end{tabular}

Table 2: Sensitivity Index for HIV and Malaria.

$$
S=\frac{\partial R_{0}}{\partial P} * \frac{P}{R_{0}}
$$

where $\mathrm{P}$ is the parameter of interest.

The sensitivity index is a local estimate of the best way to reduce $R_{0}$. The larger magnitude of the sensitivity index, the more "sensitive" $R_{0}$ is with respect to that parameter. For example if we know $R_{0}$ is dominated by HIV infection, then a 10 percent decrease in the transmission rate corresponds, roughly, to a 10 percent decrease in $R_{0}$. However a 10 percent decrease in the death rate corresponds to a 7 percent increase in $R_{0}$ for Malawi and an 8 percent increase for SubSaharan Africa. Then we are interested in the indices with the largest magnitude, thus if $R_{0}$ is dominated by $R_{O H}$, we want to control $\beta_{H}$. On the other hand, if $R_{0}$ is dominated by $R_{0 M}$, then we want to control $\beta_{V M}, \beta_{M V}$, or $\mu_{V}$.

\section{Conclusion}

A model for the co-infection of HIV and malaria was constructed and analyzed. We started with a simple system of six equations which we reduced to four. We observed it was not necessary to explicitly model the vector population to capture the dynamics of co-infection. Although there is an increase in mortality due to coinfection, this increase is not pronounced until the amplification factor is approximately 25 . In fact, if we assume there is no additional infectivity due to co-infection, the increased mortality is only 3 percent. However, the mortality nearly doubles when the increased infectivity is 30 . The biological integrations between the malaria parasite and HIV are not fully understood, but it is conceivable that the parasite or viral load can increase by an order of magnitude due to co-infection. Future studies should include fitting parameters to data. An investigation of the co-infection at a cellular level would also be interesting. In our framework we did not include treatment for simplicity, but treatment is a major component of any approach to a solution of the HIV and malaria epidemics.

\section{A Calculation of $\boldsymbol{R}$}

The next generation operator method is a systematic way to calculate $R_{0}$ [11]. $R_{0}$ is defined as the spectral radius of the next generation matrix. First we separate the classes into two groups, infectious and non-infectious. Vector $\mathrm{f}$ is composed of the new infection terms of the infectious classes.

$$
F=\left[\begin{array}{l}
\beta_{V M} I_{V} S \\
\frac{\beta_{V}}{N} \\
\frac{\beta_{H} I_{H} S+\rho_{2} \beta_{H} I_{H M} S}{0} \\
\frac{\beta_{M V} I_{M} V+\rho_{4} \beta_{M V} I_{H M} V}{N}
\end{array}\right]
$$

The vector $\mathcal{V}$ is composed of the remaining terms of the infectious classes.

$$
v=\left[\begin{array}{c}
\frac{\beta_{H} I_{H} I_{M}+\rho_{1} \beta_{H} I_{H M} I_{M}}{N}+\mu_{M} I_{M}+\gamma I_{M}+\alpha I_{M} \\
\frac{\rho_{3} \beta_{V M} I_{H} I_{V}}{N_{V}}+\mu_{H} I_{H}-k \gamma I_{H M}+\alpha I_{H} \\
-\frac{\beta_{H} I_{H} I_{M}+\rho_{1} \beta_{H} I_{H M} I_{M}}{N}-\frac{\rho_{3} \beta_{V M} I_{H} I_{V}}{N_{V}}+\mu_{H M} I_{H M}+k \gamma I_{H M}+\alpha I_{H M}
\end{array}\right]
$$

Fand Vare the Jacobians of $\mathrm{f}$ and $\mathrm{V}$ with respect to the infectious classes, respectively. Then the next generation matrix is defined as $V^{-1} F$ evaluated at the DFE, and $R_{0}$ is the dominant eigenvalue of this matrix (Table 2).

$$
V^{-1} F=\left[\begin{array}{cccc}
0 & 0 & 0 & \frac{\beta_{V M} \wedge}{\alpha N_{V} \mu_{V}} \\
0 & \frac{\beta_{H}}{\mu_{H}+\alpha} & \frac{\beta_{H} k \gamma}{\left(\mu_{H}+\alpha\right)\left(\mu_{H M}+k \gamma+\alpha\right)} & 0 \\
0 & 0 & 0 & 0 \\
\frac{\beta_{M V} N_{V} \alpha}{\wedge\left(\mu_{M}+\gamma+\alpha\right)} & 0 & \frac{\rho_{4} \beta_{M V} N_{V} \alpha}{\wedge\left(\mu_{H M}+k \gamma+\alpha\right)} & 0
\end{array}\right]
$$

For the Reduced Model we have

$$
\begin{gathered}
F=\left[\begin{array}{c} 
\\
\frac{\beta_{V M} \beta_{M V}\left(I_{M}+\rho_{4} I_{H M}\right) S}{\beta_{M V} I_{M}+\rho_{4} \beta_{M V} I_{H M}+\mu_{V} N} \\
\frac{\beta_{H} I_{H} S+\rho_{2} \beta_{H} I_{H M} S}{N} \\
0
\end{array}\right] \\
v=\left[\begin{array}{c}
\frac{\beta_{H} I_{H} I_{M}+\rho_{1} \beta_{H} I_{H M} I_{M}}{N}+\mu_{M} I_{M}+\gamma I_{M}+\alpha I_{M} \\
\frac{\rho_{3} \beta_{V M} I_{M} \beta_{M V}\left(I_{M}+\rho_{4} I_{H M}\right)}{\beta_{M V} I_{M}+\rho_{4} \beta_{M V} I_{H M}+\mu_{V} N}+\mu_{H} I_{H}-k \gamma I_{H M}+\alpha I_{H} \\
-\frac{\beta_{H} I_{H} I_{M}+\rho_{1} \beta_{H} I_{H M} I_{M}}{N}-\frac{\rho_{3} \beta_{V M} I_{H} \beta_{M V}\left(I_{M}+\rho_{4} I_{H M}\right)}{\beta_{M V} I_{M}+\rho_{4} \beta_{M V} I_{H M}+\mu_{V} N}+\mu_{H M} I_{H M}+k \gamma I_{H M}+\alpha I_{H M}
\end{array}\right]
\end{gathered}
$$

Then the next generation matrix is

$$
V^{-1} F=\left[\begin{array}{ccc}
\frac{\beta_{V M} \beta_{M V}}{\mu_{V}\left(\mu_{M}+\gamma+\alpha\right)} & 0 & \frac{\beta_{V M} \beta_{M V} \rho_{4}}{\mu_{V}\left(\mu_{H M}+k \gamma+\alpha\right)} \\
0 & \frac{\beta_{H}}{\left(\mu_{H}+\alpha\right)} & \frac{\beta_{H} k \gamma}{\left(\mu_{H}+\alpha\right)\left(\mu_{H M}+k \gamma+\alpha\right)}+\frac{\rho_{2} \beta_{H}}{\mu_{H M}+k \gamma+\alpha} \\
0 & 0 & 0
\end{array}\right]
$$

\section{References}

1. Castillo-Chavez C, Levin SA, Shoemaker CA (1987) Mathematical Approaches to Problems in Resource Management and Epidemiology, New Youk, 220-248. 
Citation: Barley K, Murillo D, Roudenko S, Tameru AM, Tatum S (2012) A Mathematical Model of HIV and Malaria Co-Infection in Sub-Saharan Africa. J AIDS Clinic Res 3:173. doi:10.4172/2155-6113.1000173

Page 7 of 7

2. http://www.hivinsite.com/

3. Ross R (1911) The Prevention of Malaria. London.

4. Ross R (1928) Studies on Malaria. London

5. Chitnis N, Cushing JM, Hyman JM (2006) Bifurcation Analysis of a Mathematical Model for Malaria Transmission. SIAM J Appl Math 67: 24-45.

6. Abu-Raddad LJ, Patnaik P, Kublin JG (2006) Dual infection with HIV and Malaria fuels the spread of both diseases in Sub-Saharan Africa. Science 314: 1603-1606.

7. Center for Disease Control (CDC) (2007).
8. van den Driessche P, Watmough J (2002) Reproduction Numbers and Sub-Threshold Endemic Equilibria for Compartmental Models of Disease Transmission. Math Biosci 180: 29-48.

9. Brauer F, Castillo-Chavez C (2000) Mathematical Models in Population Biology and Epidemiology, Springer, New York.

10. Arriola LM, Hyman JM (2007) Being Sensitive to Uncertainty, Journal Computing Science and Engineering 9: 10 - 20.

11. Corbett EL, Steketee RW, ter Kuile FO, Latif AS, Kamali A, et al. (2002) HIV-1/AIDS and the Control of other Infectious Diseases in Africa. Lancet 359: 2177-2187.

12. The World Health Organization (WHO). 